\title{
A Reliability Study on Green InGaN-GaN Light-Emitting Diodes
}

\author{
Z. L. Li, P. T. Lai, Senior Member, IEEE, and H. W. Choi, Senior Member, IEEE
}

\begin{abstract}
In this letter, the reliability of green InGaN-GaN light-emitting diodes (LEDs) has been analyzed by correlating the defect density of wafers with various device parameters, including leakage current, $1 / f$ noise, and degradation rate. It was found that as the wavelength of green LEDs increases from 520 to $550 \mathrm{~nm}$ by increasing the indium content in the quantum wells, the defect density also increases, thus leading to larger leakage current, enhanced noise magnitude, and shortened device lifetime.
\end{abstract}

Index Terms-Light-emitting diode (LED), reliability.

\section{INTRODUCTION}

$\mathbf{T}$ HE development of phosphor-free white light-emitting diodes (LEDs) relies on the availability of high-quality green LEDs at longer wavelengths. LEDs with wavelength centered at around $555 \mathrm{~nm}$ are particularly important in high-brightness RGB-based white lighting systems, coinciding with the peak photopic response of human eyes. However, it is challenging to grow InGaN-GaN LED structures for emission at longer wavelengths due to the difficulty associated with the incorporation of indium (In). This difficulty originates from the strain condition in the quantum wells (QWs), which limits the extent of In incorporation. Higher In content in a well leads to higher compressive strain in the layer, resulting in inefficient In incorporation [1].

On the other hand, higher In composition is essential to realize longer wavelength emission; it also becomes a threat to the device performance and reliability. First, the higher In content in the multiquantum-wells (MQWs) can introduce large piezoelectric field induced by the large strain. This piezoelectric field reduces the overlap of the electron and hole wave functions, and thus results in reduced internal quantum efficiency in green LEDs [2]. Second, the large lattice mismatch can give rise to high defect concentration in the structure in the form of dislocations, which result in degraded crystal quality. As a result, the higher dislocation density of green LED introduced by In incorporation can often deteriorate its performance and reliability.

In this letter, we report on the study of reliability of green InGaN-GaN LEDs by correlating the defect concentration in wafers of wavelength ranging from 520 to $550 \mathrm{~nm}$ with their low-frequency noise spectrum and 1000-h lifetime data. Surface scan by atomic force microscopy (AFM) is used to quantify the

Manuscript received April 09, 2009; revised June 12, 2009. Current version published September 18, 2009.

The authors are with the Department of Electrical and Electronic Engineering, The University of Hong Kong, Hong Kong, Hong Kong (e-mail: hwchoi@hku. hk).

Color versions of one or more of the figures in this letter are available online at http://ieeexplore.ieeee.org.

Digital Object Identifier 10.1109/LPT.2009.2028155 exposed defects after reactive ion etching (RIE) to compare the defect density of the wafers. The electrical properties and optical performances of the devices are evaluated. The devices are subsequently subjected to a 1000 -h continuous lifetime test. We attempt to explain the observed phenomenon in terms of carrier dynamics.

\section{EXPERIMENTAL DETAILS}

The structure of the LED wafers has a GaN buffer layer grown on c-plane sapphire substrate, followed by an n-type GaN layer, active layers with $\mathrm{InGaN}-\mathrm{GaN}$ as quantum well, and capped with a top p-type GaN contact. The emission wavelengths of the wafers are approximately 520, 540, and $550 \mathrm{~nm}$, respectively, obtained by varying the In content from 0.25 to 0.30 . Details of the growth will be reported elsewhere. The LEDs were fabricated by a standard microfabrication process beginning with the deposition of a semitransparent current-spreading layer composed of $\mathrm{Ni}-\mathrm{Au}(10 \mathrm{~nm} / 10 \mathrm{~nm})$ by e-beam evaporation, followed by annealing in an oxygen environment at a temperature of $550{ }^{\circ} \mathrm{C}$ for $5 \mathrm{~min}$. A $500 \times 500 \mu \mathrm{m}^{2}$ emission active area was defined by photolithography and dry etched using a chemistry comprising $\mathrm{CHF}_{3}$ and $\mathrm{Ar}$ to expose the $\mathrm{n}$-contact regions. Photolithography was again carried out to define the p-pad and n-pad areas. A bilayer of $\mathrm{Ti}-\mathrm{Al}(50 \mathrm{~nm} / 350 \mathrm{~nm})$ was deposited as p-pads and n-pads, and then annealed in $\mathrm{N}_{2}$ at $350{ }^{\circ} \mathrm{C}$ for $1 \mathrm{~min}$. The chips were diced by laser micromachining and packaged into TO-cans. After wire bonding, a silicone encapsulant was applied to protect the devices.

The defect densities of the LED structures were estimated by an AFM using a Seiko Instruments Nanopics system, after defect exposure by a plasma treatment similar to the mesa etch. The electrical properties, including the current-voltage $(I-V)$ characteristics and leakage current of three packaged devices, were measured with an HP 4156A precision semiconductor parameter analyzer, while the $1 / f$ noise spectra were measured by a BTA 9603 FET noise analyzer. The optical performances of the devices were evaluated with an Ocean Optics HR2000 fiber-coupled spectrometer. The devices, biased at $20 \mathrm{~mA}$, were subsequently subjected to a 1000 -h continuous stress test at room temperature.

\section{RESULTS AND DISCUSSION}

The AFM surface scans in Fig. 1 are used for defect estimation of the LED wafers. Defects propagate from the site where they are generated, forming 2-D dislocations. These defects include those generated at the $\mathrm{GaN}$-sapphire interface due to lattice mismatch and also those generated within the MQWs due to strain. Since dislocations terminate at an interface as voids, counting the number of such voids within unit area provides 

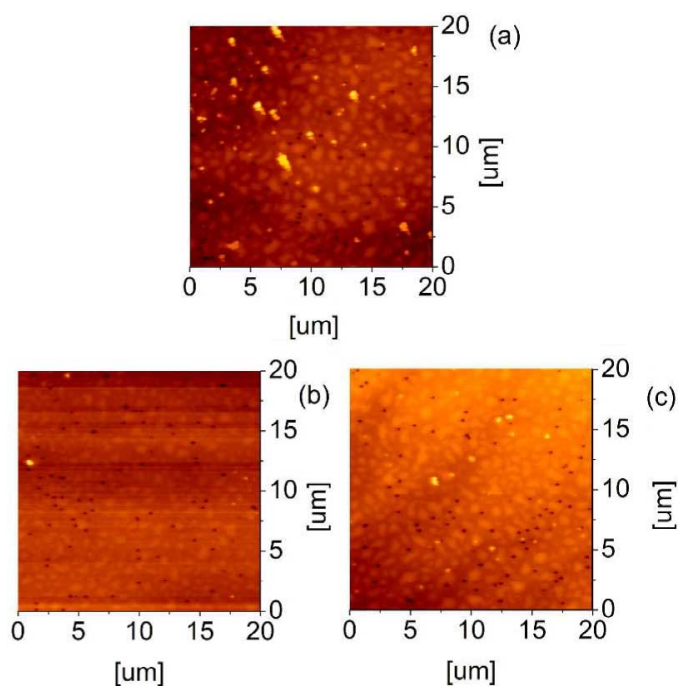

Fig. 1. $20 \times 20 \mathrm{~mm}$ AFM surface scan of the (a) 520-, (b) 540-, and (c) $550-\mathrm{nm}$ InGaN-GaN LED wafers.

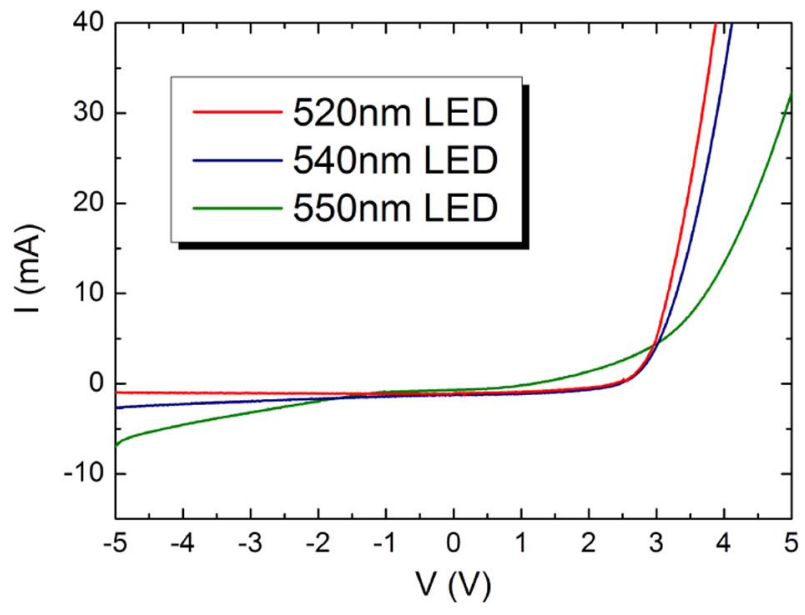

Fig. 2. $I-V$ characteristics of the three green LEDs.

data on the dislocation density. Nevertheless, such voids are of subnanometer dimension. In order to "visualize" these features, they can be enlarged by a plasma treatment. By etching away $50 \mathrm{~nm}$ of p-type GaN from the surface of the wafer, the defects are intentionally enlarged due to preferential etching of recessed areas [3], thereby resulting in the formation of $\mathrm{V}$-shaped etch pits. Etch pits are readily revealed by AFM scanning of the plasma-treated surfaces. The dislocation density of the wafers can be thus estimated from the AFM scan, making it an accurate and convenient method for determining defect density. In the $20 \mu \mathrm{m} \times 20 \mu \mathrm{m}$ surface scans shown in Fig. 1, the dark dots represent these etch pits. It is estimated that the defect density of the $520-, 540-$, and 550-nm wafers is about $1.40 \times 10^{7}, 1.75 \times 10^{7}$, and $2.22 \times 10^{7} / \mathrm{cm}^{2}$, respectively. These numbers tally well with the prediction that In incorporation leads to higher defect density.

The electrical characteristics of the LED devices fabricated from these wafers are measured. The $I-V$ characteristics of the three LEDs are presented in Fig. 2. The leakage currents of the devices have been extracted at a reverse bias voltage of $4 \mathrm{~V}$.

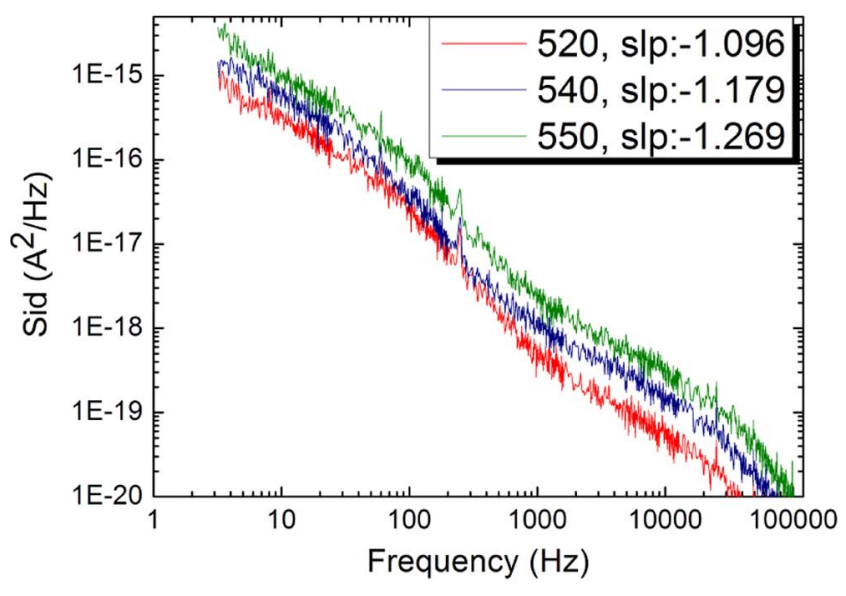

Fig. 3. $1 / f$ noise spectrum of the green LEDs.

As the wavelength of the LEDs increases, the leakage current also increases from $0.99 \mathrm{~mA}$ in the 520-nm LED to $2.29 \mathrm{~mA}$ in the 540-nm LED and $4.45 \mathrm{~mA}$ in the 550-nm LED. The slope of the linear region of the $I-V$ curve (in the forward bias region) has been interpolated to determine the static resistance of the devices. As evident from the $I-V$ plot, the slope increases as the wavelength increases. The extracted static resistances are 23.5, 33.3, and 52.6 $\Omega$ for the 520-, 540-, and 550-nm LEDs, respectively. This consistent trend in the leakage current and the static resistance can be linked to the increasingly higher defect concentration as the In incorporation increases. The dislocations act as vertical current leakage pathway, while the defects lead to carrier scattering and hence higher resistance. To verify this further, additional experiments are conducted to study the carrier properties.

Since low-frequency noise is closely related to the presence of impurities and defects in semiconductors, it can more accurately represent the internal crystal quality than other electrical parameters [4]. The noise spectra of the three LEDs are measured and plotted in Fig. 3. The system floor calibration is conducted for dc bias calculation and $1 / f$ noise correction. The range of frequency measured is between 3.15 and $105 \mathrm{~Hz}$. The slope can be extracted by the supplied software automatically. In the figure, it is observed that both the magnitude and slope of 520-nm LED are lower than the 540- and 550-nm LED.

In an LED structure, the current spectral density can be expressed by the following equation [5]:

$$
\begin{gathered}
S_{I}(f)=\frac{\alpha q I_{D}}{f \tau}+\left(\frac{q n_{1} A}{I_{0}}\right)^{2} \\
B \frac{k T N_{t}}{\ln \left(\tau_{2} / \tau_{1}\right)} \frac{1}{f} I_{R}^{2}
\end{gathered}
$$

where

$$
n_{1}=n_{i} \exp \left(\frac{E_{t}-E_{i}}{k T}\right) .
$$

The first term of (1) represents the near-equilibrium steadystate condition, whereby there is no net accumulation of charges and the generation rate is equal to the recombination rate. The variable $\alpha$ is the Hooge parameter [6], which depends on the 


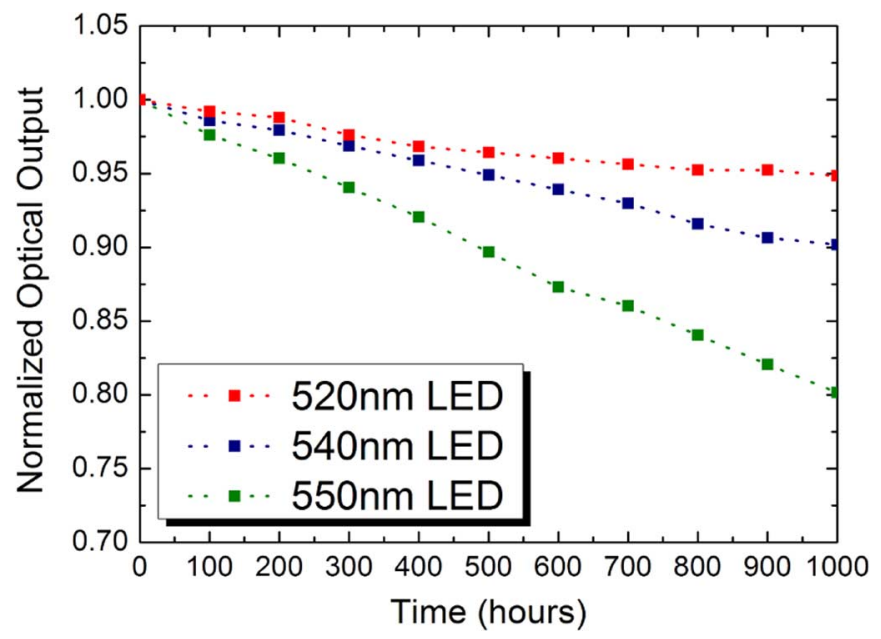

Fig. 4. 1000-h lifetime degradation test of the green LEDs.

quality of the crystal [7] and the scattering mechanisms. A larger $\alpha$ value generally implies poorer crystal quality, and increases the magnitude of the noise spectrum. $I_{D}$ is the current through the device; $\tau$ is the lifetime of minority carrier; $N_{t}$ is the trap density, which can increase the magnitude of the noise spectrum when the trap density increases; and the other terms have their usual meanings. The measured $1 / f$ noise spectrum thus indicates that the crystal quality deteriorates as the emission wavelength increases from 520 to $550 \mathrm{~nm}$ due to the increased noise magnitude, thus supporting the postulation of enhanced defect density with increasing In incorporation. Apart from the noise magnitude, it is also observed that the magnitude of the slope of the noise spectrum increases with increasing wavelength. This may be explained in terms of occupation of traps within the semiconductor material. As defect density in the InGaN-GaN layers increases, transitions between traps become more frequent, leading to a reduction in electron mean free time. Therefore, as frequency increases, these trapped electrons cannot respond quickly enough and the number of free electron in the crystal decreases, which subsequently leads to a decrease in the current spectral density at that particular frequency. With more defects in the crystal, the spectrum decreases at a faster rate, corresponding to an increase in the slope, which is consistent with our measured data.

The results can also be correlated to the lifetime of the devices. Fig. 4 shows the results of a 1000-h reliability test, whereby a constant bias current of $20 \mathrm{~mA}$ was applied to the devices. While the 520-nm LED maintains 95\% of its initial optical power after $1000 \mathrm{~h}$, the number drops to $91 \%$ and $82 \%$ for the 540- and 550-nm LEDs, respectively. Assuming linearity, the L70 lifetime (L70 lifetime is the time to 70\% lumen maintenance, as proposed by ASSIST [8]) for our 520-, 540-, and 550-nm LEDs is 6000, 3750, and $1667 \mathrm{~h}$, respectively.
Green LEDs with increasing wavelength are therefore expected to have increasingly shorter lifetime.

Despite the attractiveness of deep green LEDs in terms of photopic response, there are many other issues to be considered. As the In content in the QW regions are increased, a significant increase in defect density results. These defects act as nonradiative recombination centers, degrading optical performance. However, there are further side effects. These defects serve as vertical conduction pathways, creating large leakage currents, which subsequently affect the device lifetime. It is therefore pertinent to reduce the defect densities before longer wavelength wafers become feasible. One possible approach is to grow these epilayers on bulk GaN substrates. Based on our experimental data, it is deduced that the lifetime of LEDs can be predicted from their electrical parameters, particularly from a comparative study of their noise spectra.

\section{CONCLUSION}

In summary, the role of In incorporation in green InGaN-GaN LED has been investigated. While higher In concentration in the QW produces LEDs with longer wavelength (particularly, wavelengths around $550 \mathrm{~nm}$ with high photopic response for phosphor-free white LEDs), additional defects are introduced into the devices due to the strain induced. These defects play multiple roles in affecting device performance, including acting as nonradiative recombination centers and leakage pathways for vertical conduction. Consequently, the reliability and lifetime of devices are deteriorated. We propose the use of low-frequency noise for the prediction of device lifetime, as it is a tool that is sensitive to defect density in the material.

\section{REFERENCES}

[1] C. F. Huang, T. Y. Tang, J. J. Huang, W. Y. Shiao, C. C. Yang, C. W. Hsu, and L. C. Chen, "Prestrained effect on the emission properties of InGaN/GaN quantum-well structures," Appl. Phys. Lett., vol. 89, pp. 051913-1-051913-3, 2006.

[2] R. D. Dupuis, J. B. Limb, J. P. Liu, J. H. Ryou, C. Home, and D. Yoo, "InGaN MQW green LEDs using p-InGaN and p-InGaN/p-GaN superlattices as p-type layers," Proc. SPIE, vol. 6894, pp. 68941D1-68941D-8, 2008.

[3] H. W. Choi, C. Liu, M. G. Cheong, J. Zhang, and S. J. Chua, "Exposure of defects in GaN by plasma etching," Appl. Phys. A, Solids Surf., vol. 80, pp. 405-407, 2005.

[4] F. N. Hooge, T. G. M. Kleinpenning, and L. K. J. Vandamme, "Experimental studies on 1 / $f$ noise," Rep. Progr. Phys., vol. 44, pp. 479-532, 1981.

[5] J. Hu, L. Du, Y. Q. Zhang, J. L. Bao, and J. Zhou, "Noise as a representation for reliability of light emitting diode," Acta Phys. Sin., vol. 55, pp. 1384-1389, 2006.

[6] F. N. Hooge, "1/ $f$ noise sources," IEEE Trans. Electron. Devices, vol. 41, no. 11, pp. 1926-1935, Nov. 1994.

[7] A. Balandin, K. L. Wang, A. Svizhenko, and S. Bandyopadhyay, "The fundamental $1 / f$ noise and the hooge parameter in semiconductor quantum wires," IEEE Trans. Electron. Devices, vol. 46, no. 6, pp. 1240-1244, Nov. 1999.

[8] "LED Life for General Lighting: Definition of Life" Alliance for SolidState Illumination Systems and Technologies, 2005. 